\title{
Conservación
}

\section{Primeros registros de 4 especies de felinos en el sur de Puebla, México}

\author{
First records of 4 felid species in southern Puebla, Mexico \\ Verónica Farías $^{\mathrm{a}, *}$, Oswaldo Téllez ${ }^{\mathrm{a}}$, Francisco Botello ${ }^{\mathrm{b}, \mathrm{c}}$, Omar Hernández $^{\mathrm{c}}$, \\ Jessica Berruecos ${ }^{a}$, Saúl J. Olivares ${ }^{\mathrm{d}}$ y Julio C. Hernández ${ }^{\mathrm{a}}$ \\ a Laboratorio de Recursos Naturales, Unidad de Biología, Tecnología y Prototipos, Facultad de Estudios Superiores Iztacala, \\ Universidad Nacional Autónoma de México, Avenida de Los Barrios 1, Los Reyes Iztacala, 54090 Tlalnepantla de Baz, Estado de México, México \\ ${ }^{\mathrm{b}}$ Departamento de Zoología, Instituto de Biología, Universidad Nacional Autónoma de México, Apartado postal 70-153, 04510, México, D. F., México \\ ${ }^{\mathrm{c}}$ Conservación Biológica y Desarrollo Social, A. C., Calle Nueve 52 interior 4, Colonia Espartaco, Coyoacán, 04870, México, D. F., México \\ d Comisariado Ejidal 2012-2014, San José Axuxco, 75975 Municipio de San José Miahuatlán, Puebla, México
}

Recibido el 9 de octubre de 2014; aceptado el 24 de junio de 2015

Disponible en Internet el 5 de noviembre de 2015

\begin{abstract}
Resumen
Se presentan los primeros registros de margay (Leopardus wiedii), gato montés (Lynx rufus), puma (Puma concolor) y jaguarundi (Puma yagouaroundi) en el sur del estado de Puebla. El trabajo de campo fue parte de una monitorización participativa que incluyó a las autoridades civiles del ejido. Se colocaron 11 estaciones de cámaras-trampa digitales que funcionaron del 18 de diciembre de 2012 al 18 de febrero de 2014. Con un esfuerzo de muestreo de 2,669 días-trampa y dentro del conjunto de registros obtenidos, se pudieron registrar por primera vez para la región 4 especies de felinos mediante 3 registros de L. wiedii, 26 de L. rufus, 7 de P. concolor y 4 de P. yagouaroundi. Fue posible con estos registros documentar la presencia de una hembra adulta de $P$. concolor con sus 2 crías. Los rangos de distribución conocidos de $L$. wiedii y $P$. yagouaroundi aumentaron en el centro de México. Las especies identificadas coexisten en la selva baja caducifolia del territorio ejidal de San José Axuxco, Puebla, dentro de los límites de la Reserva de la Biosfera Tehuacán-Cuicatlán. La localidad debe de considerarse prioritaria para la conservación de las poblaciones de las especies de felinos amenazados en la región.

Derechos Reservados (C) 2015 Universidad Nacional Autónoma de México, Instituto de Biología. Este es un artículo de acceso abierto distribuido bajo los términos de la Licencia Creative Commons CC BY-NC-ND 4.0.
\end{abstract}

Palabras clave: Cámara-trampa; Especie amenazada; Monitorización participativa; Reserva de la Biosfera Tehuacán-Cuicatlán

\section{Abstract}

The first records of margay (Leopardus wiedii), bobcat (Lynx rufus), cougar (Puma concolor), and yaguarundi (Puma yagouaroundi) in the southern state of Puebla are presented. Field work was achieved by participative monitoring in collaboration with local authorities. A total of 11 stations of digital camera traps were set up that worked from December 18th, 2012 thru February 19th, 2014. With a sampling effort of 2,669 trap-days and with the presence data obtained it was possible to document for the first time the presence of 4 felid species for the region through 3 records of L. wiedii, 26 of L. rufus, 7 of P. concolor, and 4 of P. yagouaroundi. It was also possible to document the presence of one adult female of $P$. concolor with her 2 kittens. The known distribution ranges of $L$. wiedii and $P$. yagouaroundi were enlarged for central México. The identified species coexist in the tropical dry forest of San José Axuxco within the limits of Tehuacán-Cuicatlán biosphere reserve. The locality is potentially prioritary for the conservation of populations of endangered felids in the region.

All Rights Reserved (C) 2015 Universidad Nacional Autónoma de México, Instituto de Biología. This is an open access item distributed under the Creative Commons CC License BY-NC-ND 4.0.

Keywords: Camera trap; Endangered species; Participatory monitoring; Biosphere Reserve Tehuacán-Cuicatlán

\footnotetext{
* Autora para correspondencia.

Correo electrónico: v.farias@ campus.iztacala.unam.mx (V. Farías).

La revisión por pares es responsabilidad de la Universidad Nacional Autónoma de México.
} 


\section{Introducción}

La identificación de localidades donde aún habitan los felinos silvestres en México es prioritaria para ubicar dónde se deben prevenir y atenuar los riesgos que amenazan a sus poblaciones (Almazán-Catalán et al., 2013; Briones-Salas, Lavariega y LiraTorres, 2012). En el estado de Puebla habitan al menos 5 de las 6 especies de la familia Felidae que se distribuyen en nuestro país (Aranda, 2012; Ramírez-Bravo, Bravo-Carrete, HernándezSantín, Schinkel-Brault y Kinnear, 2010; Ramírez-Pulido, González-Ruiz y Genoways, 2005); sin embargo, son contados los registros de presencia que documentan ejemplares en colecciones científicas, observación directa, evidencia fotográfica o identificación de huellas, excrementos y pieles (Ramírez-Bravo, Bravo-Carreteet al., 2010; Tewes y Everett, 1982). RamírezPulido et al. (2005) presentaron una revisión detallada sobre registros de mamíferos carnívoros para el estado de Puebla y corroboraron la presencia de 4 especies de felinos. Los autores identificaron un excremento de puma (Puma concolor), encontraron un registro de gato montés (Lynx rufus) que data de finales del siglo XIX (Ferrari-Pérez, 1886) y revisaron 2 ejemplares de jaguarundi (Puma yagouaroundi) y 2 cráneos de margay (Leopardus wiedii) depositados en colecciones científicas. Ramírez-Bravo, Bravo-Carrete et al. (2010), y Ramírez-Bravo, Schinkel-Brault y Hernández-Santín (2010) documentaron la presencia del ocelote (Leopardus pardalis) en el norte del estado.

En México es común encontrar felinos simpátricos debido a que existen condiciones para la presencia de especies de origen neártico y neotropical (Johnson et al., 2006) y el estado de Puebla se encuentra en la zona de transición mexicana (Escalante, Rodríguez y Morrone, 2005). Cuando el hábitat es capaz de cubrir las demandas de territorio y abundancia de presas, $2 \mathrm{o}$ más especies de felinos silvestres pueden coexistir permitiendo la repartición de recursos a través de mecanismos como la segregación espacial y/o temporal y/o la variación en el porcentaje de consumo de las presas compartidas (Di Bitetti, de Angelo, di Blanco y Paviolo, 2010; Emmons, 1987; Foster et al., 2013; Harmsen, Foster, Silver, Ostro y Doncaster, 2009; MonroyVilchis, Rodríguez-Soto, Zarco-González y Urios, 2009; Núñez, Miller y Lindzey, 2000). Los grandes felinos silvestres de México, el jaguar (Panthera onca) y el puma, requieren grandes extensiones para establecer su ámbito hogareño y encontrar suficiente alimento (Briones-Salas et al., 2012; Núñez et al., 2000). El jaguar es el único felino que no ha sido documentado para el estado de Puebla y se encuentra en peligro de extinción (Ramírez-Bravo, Bravo-Carrete et al., 2010; RamírezBravo, Schinkel-Braut et al., 2010; Ramírez-Pulido et al., 2005; Semarnat, 2010). El ocelote es subordinado ante felinos de mayor tamaño en la competencia intragremial y también está en peligro (Aranda, 2005b; Di Bitetti et al., 2010; Emmons, 1987; Semarnat, 2010). El jaguarundi es una especie que tiene presencia en densidades bajas y, aunque puede alimentarse de roedores en ecotonos con cultivos, sus poblaciones necesitan cobertura vegetal en buen estado y cercana a fuentes de agua, por lo que se encuentra amenazado por la transformación del hábitat (Almazán-Catalán et al., 2013; Aranda, 2005a; Oliveira, 1998a; Reid, 1997; Semarnat, 2010). El gato montés es el felino de tamaño mediano con mayor distribución en México y ocupa gran variedad de ambientes; aunque es más común en matorrales áridos y bosques templados son pocos los lugares donde coexiste con el ocelote y/o el jaguarundi (Aranda, 2012). El margay o tigrillo es el más pequeño y elusivo de los felinos en México, sus hábitos arborícolas o semiarborícolas lo convierten en la especie menos conocida y estudiada, es sensible a la transformación del hábitat y se encuentra en peligro de extinción (Aranda, 2005b; Oliveira, 1998b; Reid, 1997; Semarnat, 2010). Debido al reducido número de registros históricos de felinos silvestres para Puebla, este trabajo contribuye a documentar los primeros registros de presencia de $L$. wiedii, $L$. rufus, $P$. concolor y $P$. yagouaroundi en el sur del estado (fig. 1), dentro de los límites de la Reserva de la Biosfera Tehuacán-Cuicatlán (RBTC).

\section{Materiales y métodos}

El ejido de San José Axuxco se encuentra al sur del estado de Puebla en las coordenadas geográficas $18^{\circ} 13^{\prime} 52.2^{\prime \prime} \mathrm{N}$ 97 $12^{\prime} 26.3^{\prime \prime} \mathrm{O}$, altitud $959 \mathrm{~m} \mathrm{snm}$; pertenece al municipio de San José Miahuatlán que colinda al oeste con el municipio de Caltepec, al norte con San Gabriel Chilac, Altepexi y Zinacatepec, al este con Coxcatlán y al sur con el estado de Oaxaca (fig. 2). Parte del territorio de San José Axuxco se encuentra dentro del polígono de la RBTC, donde los ejidatarios protegen el hábitat para la vida silvestre. En el territorio ejidal que se encuentra colindando con la reserva, la principal actividad económica es el cultivo de la caña de azúcar y en menor medida los cultivos de maíz y frijol de subsistencia. La crianza de ganado doméstico es a baja escala y se aprecian caprinos, vacunos y equinos. El clima es semiárido con lluvias en verano con una precipitación promedio anual de $300 \mathrm{~mm}$ y temperatura promedio anual de $22^{\circ} \mathrm{C}$. La vegetación en los territorios ejidales que se encuentran dentro de los límites de la RBTC es selva baja caducifolia con cactáceas columnares de los géneros Cephalocereus, Myrtillocactus, Neobuxbaumia y Pachycereus, entre otros, como dominante fisionómico (Rzedowski, 1978; Valiente-Banuet et al., 2000), y árboles y arbustos de los géneros Bursera, Castela, Ceiba, Ficus, Fouqueria, Parkinsonia y Ziziphus (Rzedowski, 1978; Valiente-Banuet et al., 2000).

El trabajo de campo se llevó a cabo mediante la monitorización participativa con las autoridades civiles del ejido y dando aviso oportuno a las autoridades de la reserva (Botello et al., 2013). Nuestra unidad de muestreo fueron las estaciones de fototrampeo; cada estación consistió en una cámara-trampa digital (LTL Acorn 6210, Little Acorn Outdoors, Green Bay, Wisconsin, EE. UU.) con un sensor infrarrojo que activó la cámara al detectar la presencia de un animal. Las estaciones se colocaron para detectar la presencia/ausencia de mamíferos silvestres en sitios identificados por los ejidatarios donde hallamos evidencias indirectas de presencia como huellas y excrementos.

Las estaciones se colocaron separadas por una distancia $\geq 500 \mathrm{~m}$ lineales, no se abrieron senderos para este estudio y se utilizaron los senderos ya existentes utilizados por la fauna silvestre. Cada cámara-trampa se ató en la base de una cactácea o de un árbol a una distancia de $20-40 \mathrm{~cm}$ del suelo. Se programaron para operar las $24 \mathrm{~h}$ del día y al ser activadas por el 


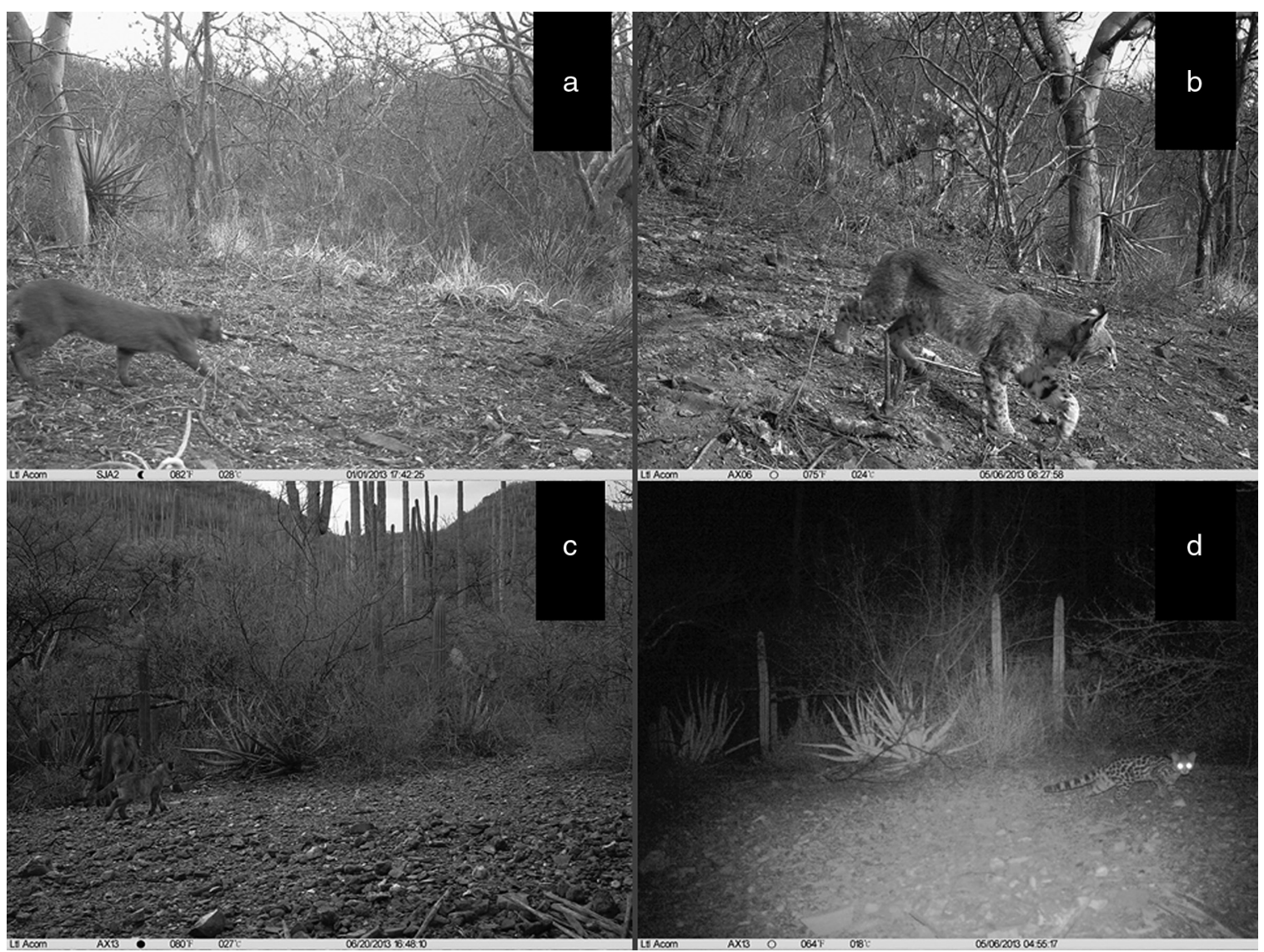

Figura 1. Registros fotográficos de: a) Puma yagouaroundi; b) Lynx rufus; c) P. concolor y d) Leopardus wiedii capturados con cámaras-trampa en el ejido de San José Axuxco, municipio de San José Miahuatlán, Puebla, en selva baja caducifolia y dentro de los límites de la Reserva de la Biosfera Tehuacán-Cuicatlán.

sensor tomar una foto de 12 megapixeles y un video de $20 \mathrm{seg}$ de duración, e imprimir la fecha y hora de captura. El periodo de intervalo entre cada evento de detección fue de 30 seg. Las cámaras se revisaron cada 5-8 semanas para obtener el material fotográfico y abastecerlas con baterías nuevas. Del 18 de diciembre de 2012 al 19 de febrero de 2013 hubo 3 estaciones operativas. Una cámara se descompuso y del 19 de febrero al 18 de abril de 2013 operaron 2 estaciones. El 18 y 19 de abril se colocaron 9 cámaras nuevas y el número de estaciones aumentó a 11 que operaron al 19 de diciembre de 2013. El 19 de diciembre se descompuso una cámara y la retiramos del campo, por lo que dejamos 10 estaciones operativas, pero el 23 de enero de 2014 se descubrió durante la revisión que 2 cámaras fueron tomadas del sitio donde se colocaron, por lo que solo obtuvimos la información de 8 estaciones del 19 de diciembre de 2013 al 23 de enero de 2014. Se colocó una nueva cámara en uno de los sitios donde fueron sustraídas y 9 estaciones operaron del 23 de enero al 18 de febrero de 2014.

Para estimar el esfuerzo de muestreo se sumó el número total de días (un día $=24 \mathrm{~h}$ ) que estuvieron funcionando las estaciones. Un día-trampa representó una estación funcionando durante las $24 \mathrm{~h}$ de un día. No se registró más de una vez a ninguna especie de felino en la misma estación de muestreo durante el mismo día. En 2 ocasiones se capturó a más de un individuo de la misma especie en el mismo registro; en este caso, se tomó como un registro con varios individuos.

\section{Resultados}

Con un esfuerzo de captura de 2,669 días-trampa se obtuvieron 40 registros de 4 especies de felinos en San José Axuxco, Puebla, durante el periodo de muestreo del 18 de diciembre de 2012 al 18 de febrero de 2014 (tabla 1). El primer felino registrado en este estudio fue un individuo de $P$. yagouaroundi el 1 de enero de 2013 a las 17:42 h (fig. 1a) en la estación 02, con un esfuerzo de 14 días-trampa (tabla 2). La coloración del pelaje del individuo correspondió a la fase de color café canelo (Aranda, 2005a; Oliveira, 1998a; Reid, 1997). El 4 de diciembre de 2013 a las 18:05 h se capturó otro individuo adulto en la estación 14, pero no se pudo distinguir la coloración del pelaje por ser una foto en tonos de grises. El tercer registro correspondió a un macho adulto con pelaje color café canelo el 1 de enero de 2014 a las 15:31 h en la estación 12 (tabla 2). El cuarto registro fue el 26 de enero de 2014 a las 8:09 h en la estación 04; se capturó la foto de un individuo también con pelaje color café canelo. Los registros de $P$. yagouaroundi de este trabajo se localizaron a una distancia lineal de $195 \mathrm{~km}$ del registro del norte de Puebla (fig. 2) en Hueytamalco documentado por Ramírez-Pulido et al. (2005), a $71 \mathrm{~km}$ del registro histórico más cercano en el centro de México en Orizaba, Veracruz, y a $65 \mathrm{~km}$ al registro más cercano en la RBTC en San José del Chilar, Oaxaca (Botello et al., 2013). 


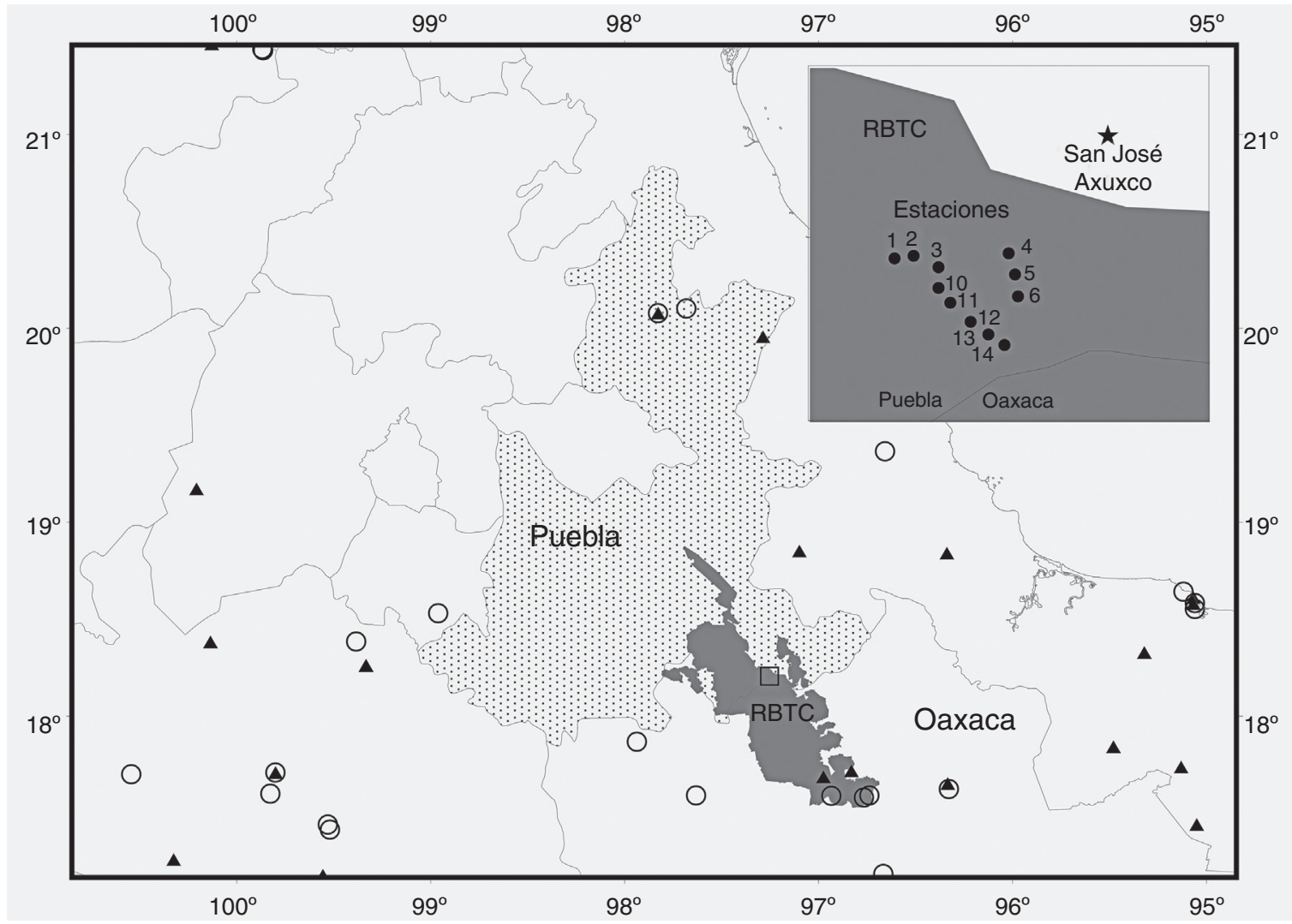

Figura 2. El ejido de San José Axuxco se encuentra en el sur de Puebla y colinda con Oaxaca. La ventana muestra el arreglo espacial de las estaciones de muestreo en el ejido y dentro de los límites de la Reserva de la Biosfera Tehuacán-Cuicatlán (RBTC). El mapa indica la ubicación geográfica de San José Axuxco (cuadrado) en relación con los registros conocidos de Leopardus wiedii (círculos) y Puma yagouaroundi (triángulos) en el centro de México.

La siguiente especie de felino en ser registrada fue L. rufus (fig. 1b) el 22 de febrero de 2013 a las 21:38 h en la estación 02, con un esfuerzo de 66 días-trampa (tabla 2). Para esta especie solo se obtuvieron registros de individuos solitarios y ocurrieron durante los meses de febrero $(\mathrm{n}=2)$, marzo (1), abril (1), mayo (3), junio (5), julio (1) agosto (4), septiembre (2), octubre (2), noviembre (1) y diciembre (1) de 2013, y enero (1) y febrero (2) de 2014. La menor parte de los registros de gato montés se colectaron en horario diurno $(8: 00-15: 59 \mathrm{~h}, \mathrm{n}=4)$, mientras que en horario nocturno (20:00-03:59 h) hubo 8 registros y en horario crepuscular 7 registros entre las $04: 00$ y $07: 59 \mathrm{~h}$ y otros 7 registros entre las 16:00 y las 19:59h. El 25 de marzo de 2013 se registró en video a un gato montés rociando con orina un tronco para dejar una marca olfativa (Aranda, 2012).
La primera fotografía de $P$. concolor se obtuvo el 20 de abril de 2013 a las 01:27 h en la estación 14, con un esfuerzo de captura de 2 días-trampa y se registró a una hembra gestante. La siguiente captura fue el 20 de junio de 2013 a las 16:48 h en la estación 13 (tabla 2), donde fue fotografiada la hembra adulta ya con sus 2 cachorros (fig. 1c). Las pintas negras en la piel y el tamaño de los cachorros permitieron estimar su edad en aproximadamente 4 a 5 semanas (Aranda, 2012; Chávez-Tovar, 2005; Currier, 1983). El 23 de junio de 2013 se registró un individuo solitario a las 04:34 h, posiblemente un macho adulto, en la estación 03 (tabla 2). El 8 de octubre de 2013 se registró a la hembra adulta con sus 2 cachorros a las 08:51 h en la estación 06 (tabla 2). Las manchas en la piel de los cachorros estaban desvanecidas, pero distinguibles y su tamaño corporal fue similar al de la hembra

Tabla 1

Frecuencia de registros y número de estaciones con registros de 4 especies de felinos en el ejido de San José Axuxco, Puebla, México, del 18 de diciembre de 2012 al 18 de febrero de 2014, con un total de 11 estaciones operativas.

\begin{tabular}{llcll}
\hline Especie & $\begin{array}{l}\text { Peso corporal } \\
(\mathrm{kg})^{*}\end{array}$ & Frecuencia de registros & $\begin{array}{l}\text { Número de estaciones } \\
\text { con registros }\end{array}$ & $\begin{array}{l}\text { Proporción de } \\
\text { estaciones con registros }\end{array}$ \\
\hline Puma concolor & $30-100$ & 7 & 6 & 0.54 \\
Lynx rufus & $5-15$ & 26 & 9 & 0.82 \\
Puma yagouaroundi & $3-9$ & 4 & 2 & 0.36 \\
Leopardus wiedii & $3-7$ & 3 & 2 & 0.18 \\
\hline
\end{tabular}

\footnotetext{
* Tomada de Aranda (2012).
} 
Tabla 2

Descripción de las estaciones con los registros de cada especie de felino y el esfuerzo de muestreo requerido para cada captura.

\begin{tabular}{|c|c|c|c|c|c|}
\hline $\begin{array}{l}\text { Nombre de la } \\
\text { estación }\end{array}$ & $\begin{array}{l}\text { Coordenadas } \\
\text { geográficas }\end{array}$ & $\begin{array}{l}\text { Altitud } \\
\text { (m snm) }\end{array}$ & $\begin{array}{l}\text { Tipo de } \\
\text { vegetación }\end{array}$ & $\begin{array}{l}\text { Fecha de } \\
\text { colocación }^{a}\end{array}$ & Felinos $^{b}$ \\
\hline 01 & $\begin{array}{l}18^{\circ} 12^{\prime} 7.60 ” \mathrm{~N} \\
-97^{\circ} 15^{\prime} 27.97^{\prime} \mathrm{O}\end{array}$ & 1,211 & $\mathrm{SBC}$ & $\begin{array}{l}18 \operatorname{dic} 2012 \\
(268)\end{array}$ & $\begin{array}{l}\text { L.rufus }(121) \\
P . \text { concolor }(265)\end{array}$ \\
\hline 02 & $\begin{array}{l}18^{\circ} 12^{\prime} 9.58^{\prime \prime} \mathrm{N} \\
-97^{\circ} 15^{\prime} 11.74 ” \mathrm{O}\end{array}$ & 1,176 & SBC & $\begin{array}{l}18 \text { dic } 2012 \\
(363)\end{array}$ & $\begin{array}{l}\text { P. yagouaroundi }(14) \\
\text { L. rufus }(66,68,134,143 \text {, } \\
152,197,199,277,347)\end{array}$ \\
\hline 03 & $\begin{array}{l}18^{\circ} 11^{\prime} 59.96 ” \mathrm{~N} \\
-97^{\circ} 14^{\prime} 50.53 ” \mathrm{O}\end{array}$ & 1,118 & SBC & $\begin{array}{l}18 \text { dic } 2012 \\
(446)\end{array}$ & $\begin{array}{l}\text { L. rufus }(97,146,249) \\
P . \text { concolor }(206)\end{array}$ \\
\hline 04 & $\begin{array}{l}18^{\circ} 12^{\prime} 12.28^{\prime \prime} \mathrm{N} \\
-97^{\circ} 13^{\prime} 50.99 ” \mathrm{O}\end{array}$ & 1,148 & SBC & $\begin{array}{l}19 \text { abr } 2013 \\
(305)\end{array}$ & $\begin{array}{l}\text { L. rufus }(107,124,157, \\
218,267) \\
\text { P. yagouaroundi }(281)\end{array}$ \\
\hline 05 & $\begin{array}{l}18^{\circ} 11^{\prime} 53.772 " \\
-97^{\circ} 13^{\prime} 45.6594 ”\end{array}$ & 1,277 & SBC & $\begin{array}{l}19 \text { abr } 2013 \\
(189)\end{array}$ & L. rufus $(100,123)$ \\
\hline 06 & $\begin{array}{l}18^{\circ} 11^{\prime} 34.91 ” \mathrm{~N} \\
-97^{\circ} 13^{\prime} 43.14 ” \mathrm{O}\end{array}$ & 1,329 & SBC & $\begin{array}{l}19 \text { abr } 2013 \\
(202)\end{array}$ & $\begin{array}{l}\text { L. rufus }(39) \\
\text { P. concolor }(64,169)\end{array}$ \\
\hline 10 & $\begin{array}{l}18^{\circ} 11^{\prime} 42.43 ” \mathrm{~N} \\
-97^{\circ} 14^{\prime} 51.11^{\prime \prime} \mathrm{O}\end{array}$ & 1,041 & SBC CC & $\begin{array}{l}18 \text { abr } 2013 \\
(167)\end{array}$ & $\begin{array}{l}\text { L. wiedii }(110) \\
\text { P. concolor }(120) \\
\text { L. rufus }(165)\end{array}$ \\
\hline 11 & $\begin{array}{l}18^{\circ} 11^{\prime} 29.9034 " \\
-97^{\circ} 14^{\prime} 40.2714 "\end{array}$ & 1,015 & $\mathrm{SBC} C \mathrm{C}$ & $\begin{array}{l}18 \text { abr } 2013 \\
(235)\end{array}$ & $\begin{array}{l}\text { No se registró ningún } \\
\text { felino }\end{array}$ \\
\hline 12 & $\begin{array}{l}18^{\circ} 11^{\prime} 13.70 ” \mathrm{~N} \\
-97^{\circ} 14^{\prime} 23.21 ” \mathrm{O}\end{array}$ & 1,019 & $\mathrm{SBC} C \mathrm{C}$ & $\begin{array}{l}18 \text { abr } 2013 \\
(306)\end{array}$ & $\begin{array}{l}\text { L. rufus }(63,173,250) \\
\text { P. yagouaroundi }(272)\end{array}$ \\
\hline 13 & $\begin{array}{l}18^{\circ} 11^{\prime} 2.94 ” \mathrm{~N} \\
--97^{\circ} 14^{\prime} 8.23^{\prime \prime} \mathrm{O}\end{array}$ & 997 & SBC CC & $\begin{array}{l}18 \text { abr } 2013 \\
(306)\end{array}$ & $\begin{array}{l}\text { L. wiedii }(17,114) \\
\text { L. rufus }(57) \\
\text { P. concolor }(63)\end{array}$ \\
\hline 14 & $\begin{array}{l}18^{\circ} 10^{\prime} 54.41^{\prime \prime} \mathrm{N} \\
-97^{\circ} 13^{\prime} 54.55^{\prime \prime} \mathrm{O}\end{array}$ & 999 & $\mathrm{SBC} C \mathrm{C}$ & $\begin{array}{l}18 \text { abr } 2013 \\
(306)\end{array}$ & $\begin{array}{l}\text { P. concolor }(2) \\
P . \text { yagouaroundi }(230)\end{array}$ \\
\hline
\end{tabular}

SBC: selva baja caducifolia; SBC CC: selva baja caducifolia con cactáceas columnares.

a En el paréntesis se indica el esfuerzo de muestreo total en días-trampa para cada estación.

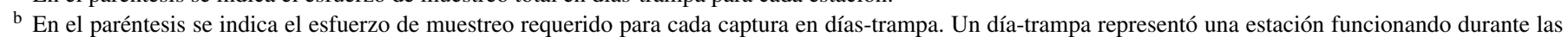
24 h de un día.

adulta, por lo que se estimó su edad entre 4 y 5 meses (Aranda, 2012; Chávez-Tovar, 2005; Currier, 1983). El 2 de enero de 2014 se registró un individuo, probablemente la hembra adulta, a las 18:36 h en la estación 10 (tabla 2). El 17 de enero de 2014 a las $11: 28 \mathrm{~h}$ se obtuvieron registros de la hembra adulta y uno de los cachorros en la estación 06. El 15 de febrero de 2014 a las 08:52 h se registró a un individuo adulto, probablemente la hembra adulta, en la estación 01.

Para $L$. wiedii se obtuvieron 2 capturas en la estación 13 (tabla 2) el 6 de mayo de 2013 a las 04:55 h (fig. 1d) y el 10 de agosto a las 00:41 h. Un tercer registro ocurrió el 23 de diciembre de 2013 a las 20:07 h en la estación 10. Los registros documentados en este trabajo se localizaron a una distancia lineal de $220 \mathrm{~km}$ de los registros en el norte de Puebla (fig. 2) en Ocotal y Olintla documentados por Ramírez-Pulido et al. (2005), de $143 \mathrm{~km}$ al registro histórico más cercano en el centro de México en Villa Emiliano Zapata, Veracruz, y de $75 \mathrm{~km}$ al registro más cercano dentro de la RBTC en Santiago Nacaltepec, Oaxaca (Botello, Illoldi-Rangel, Linaje y Sánchez-Cordero, 2006).

\section{Discusión}

Los resultados son evidencia de la presencia de las especies simpátricas $L$. rufus, $L$. wiedii, $P$. concolor y $P$. yagouaroundi, en el ejido de San José Axuxco, en el sur del estado de
Puebla, dentro de los límites de la RBTC. Los felinos son especies prioritarias para la conservación en nuestro país, y en las localidades donde varias especies coexisten es necesario estudiar los mecanismos de segregación para detectar a las especies con menor capacidad de respuesta ante las amenazas. Por ejemplo, las especies vulnerables como $L$. wiedii podrían ser excluidas por competencia interespecífica en hábitat deteriorado o transformado, o los grandes felinos como $P$. concolor podrían enfrentar presiones como la cacería ilegal y los conflictos con actividades ganaderas (Almazán-Catalán et al., 2013; Aranda, 1991; Briones-Salas et al., 2012; Di Bitetti et al., 2010). Las frecuencias de nuestros registros mostraron variación entre especies (tabla 1); sin embargo, las diferencias en el tamaño corporal entre los felinos y en el tamaño de sus ámbitos hogareños, así como sus hábitos particulares, pueden hacer variar la probabilidad de detección para cada especie por el método del fototrampeo (Di Bitetti et al., 2010; Foster y Harmsen, 2012; Kéry, 2011; Tobler, Carrillo-Percastegui, Leite-Pitman, Mares y Powell, 2008), por lo que la diferencia en las frecuencias debe también considerarse bajo este criterio. Mediante el fototrampeo se obtuvo información biológica valiosa sobre los felinos que es complementaria a la información publicada de otras investigaciones que trabajaron con patrones de actividad, uso del hábitat y segregación del nicho (Aranda, 2012; Di Bitetti et al., 2010; Iglesias et al., 2008; Lira-Torres y Briones-Salas, 2012; Monroy-Vilchis, Zarco-González, Rodríguez-Soto, Soria-Díaz y Urios, 2011; 
Valenzuela-Galván, de León-Ibarra, Lavalle-Sánchez, OrozcoLugo y Chávez, 2013).

Para el jaguarundi, las fechas de las fotocolectas fueron congruentes con la época más común de apareamiento, de enero a marzo, de acuerdo con Aranda (2005a), Oliveira (1998a) y Reid (1997). El horario de actividad estuvo en concordancia con Aranda (2005a) y Oliveira (1998a) y con investigaciones donde se registra que la especie es principalmente diurna y ocasionalmente tiene actividad crepuscular y que prefiere habitar en ecotonos entre bosques y áreas abiertas (Di Bitetti et al., 2010; Lira-Torres y Briones-Salas, 2012; Monterrubio-Rico et al., 2012). En San José Axuxco la presencia del jaguarundi puede deberse a que la selva baja caducifolia se encuentra cercana a los cañaverales que antes de ser transformados fueron pastizales nativos. Los ejidatarios han observado ocasionalmente a estos solitarios felinos buscando roedores para alimentarse en los cañaverales, y Botello et al. (2013) documentaron avistamientos de P. yagouaroundi en los años 2007 y 2010 en la zona de cañaverales del municipio de Coxcatlán, colindante con San José Axuxco. Esta información confirma que una población de jaguarundi habita en la región, lo cual es relevante para la conservación de la especie, ya que se conoce poco sobre el estatus de las poblaciones en México (Holbrook, Caso, Deyoung y Tewes, 2013).

Para L. wiedii el horario de los registros es congruente con lo documentado, en los cuales los autores suponen que es una especie nocturna cuando coexiste con el jaguarundi (Di Bitetti et al., 2010; Lira-Torres y Briones-Salas, 2012; MonroyVilchis et al., 2011; Oliveira, 1998b). Son pocos los estudios de L. wiedii en ecosistemas áridos y arbustivos, y de acuerdo con Almazán-Catalán et al. (2013), Oliveira (1998b) y ValenzuelaGalván et al. (2013) su presencia podría estar limitada a sitios con cañones y cobertura vegetal densa. Tal es el caso de San José Axuxco donde la selva baja caducifolia con cactáceas columnares provee de vegetación densa y árboles y el terreno accidentado forma cañones extensos. Botello et al. (2006) documentaron la presencia de esta especie en la región de la Cañada Oaxaqueña que forma un continuo topográfico con San José Axuxco, por lo que es posible la existencia de una población de este felino en peligro de extinción entre el norte de Oaxaca y el sur de Puebla.

Los registros de jaguarundi y de margay resultaron sobresalientes porque se incrementó el rango de distribución conocido de estas especies amenazadas (Semarnat, 2010) en el centro de México (fig. 2). También es importante mencionar que los registros de este estudio se localizaron dentro de los límites de la RBTC en territorio ejidal destinado a la protección de fauna silvestre. El método de fototrampeo permitió detectar la presencia de varios individuos de $P$. concolor durante un evento de reproducción: una hembra y sus 2 crías, y un macho adulto. Los cachorros de puma nacieron y crecieron dentro de los territorios protegidos de San José Axuxco. Es posible que la abundancia de presas potenciales como el venado cola blanca (Odocoileus virginianus) y el pecarí de collar (Pecari tajacu), así como la prohibición de la cacería de fauna silvestre, sean factores para que se puedan registrar estos ejemplares en la zona. El venado cola blanca y los pecaríes también fueron detectados por las cámaras-trampa durante el estudio en el ejido. Ramos-Robles,
Gallina y Mandujano (2013) estimaron y compararon la densidad de $O$. virginianus en 4 localidades de la RBTC con selva baja caducifolia y consideraron que en el municipio de Casablanca, mismo que colinda con los territorios de San José Axuxco, la densidad de venados $\left(2.6 \mathrm{ind} / \mathrm{km}^{2}\right)$ fue relativamente alta.

Los territorios ejidales de San José Axuxco pueden ser de gran importancia para la conservación de las poblaciones de estas especies de felinos en la región, debido a que se encuentran dentro de los límites de la RBTC y a que los pobladores locales están comprometidos con la conservación del hábitat para la vida silvestre mediante medidas como la prohibición de la cacería y la continua vigilancia. Briones-Salas et al. (2012) reconocieron que en Oaxaca las áreas comunales protegidas son una excelente estrategia de conservación para el jaguar, debido a que mantienen parte del territorio comunitario intacto y se prohíbe la cacería. Otros estudios también concluyeron que es necesario apoyar a las comunidades rurales que poseen tierras en las reservas de la biosfera para que preserven la vegetación nativa y en los alrededores de las reservas implementen actividades compatibles con la conservación (Porras-Murillo, Sarmiento-Aguilar, Naranjo y Vázquez, 2011; Valenzuela-Galván y Vázquez, 2008).

\section{Agradecimientos}

A los pobladores y a las autoridades ejidales de San José Axuxco, municipio de San José Miahuatlán, Puebla, por la participación en esta investigación y el interés genuino en salvaguardar la biodiversidad en sus tierras. A la Comisión Nacional de Áreas Naturales Protegidas, ya que a través de las autoridades de la Reserva de la Biosfera Tehuacán-Cuicatlán otorgaron las facilidades y el permiso para realizar este estudio. A la Comisión Nacional Forestal por brindar el apoyo a las autoridades civiles de San José Axuxco a través del pago de servicios ambientales para realizar recorridos de vigilancia. Asimismo, se agradece a 2 revisores anónimos las sugerencias y comentarios al manuscrito. La investigación se realizó gracias al Programa UNAM-DGAPA-PAPIIT mediante los proyectos IA200812, IN221814.

\section{Referencias}

Almazán-Catalán, J. A., Sánchez-Hernández, C., Ruíz-Gutiérrez, F., RomeroAlmaraz, M. L., Taboada-Salgado, A., Beltrán-Sánchez, E., et al. (2013). Registros adicionales de felinos del estado de Guerrero, México. Revista Mexicana de Biodiversidad, 84, 347-359.

Aranda, J. M. (1991). Wild mammal skin trade in Chiapas. En J. G. Robinson y K. H. Redford (Eds.), Neotropical wildlife use and conservation (pp. 174-177). Chicago: University of Chicago Press.

Aranda, M. (2005a). Herpailurus yagouaroundi (Lacépède, 1809). En G. Ceballos y G. Oliva (Coords.), Los mamíferos silvestres de México (pp. 358-359). México, D.F.: Comisión Nacional para el Conocimiento y Uso de la Biodiversidad, Fondo de Cultura Económica.

Aranda, M. (2005b). Leopardus wiedii (Schinz, 1821). En G. Ceballos y G. Oliva (Coords.), Los mamíferos silvestres de México (pp. 361-362). México, D.F.: Comisión Nacional para el Conocimiento y Uso de la Biodiversidad, Fondo de Cultura Económica.

Aranda, J. M. (2012). Manual para el rastreo de mamíferos silvestres de México. México, D.F.: Comisión Nacional para el Conocimiento y Uso de la Biodiversidad. 
Botello, F., Illoldi-Rangel, P., Linaje, M. y Sánchez-Cordero, V. (2006). Primer registro del tigrillo (Leopardus wiedii, Schinz 1821) y del gato montés (Lynx rufus, Kerr 1792) en la Reserva de la Biosfera de Tehuacán-Cuicatlán, Oaxaca, México. Acta Zoológica Mexicana, 22, 135-139.

Botello, F., Villaseñor, E., Guevara, L., Méndez, A., Cortés, A., Iglesias, J., et al. (2013). Registros notables del zorrillo manchado (Spilogale angustifrons) y del jaguarundi (Puma yagouaroundi) en la Reserva de la Biosfera de Tehuacán-Cuicatlán, Oaxaca, México. Revista Mexicana de Biodiversidad, 84, 713-717.

Briones-Salas, M., Lavariega, M. C. y Lira-Torres, I. (2012). Distribución actual y potencial del jaguar (Panthera onca) en Oaxaca, México. Revista Mexicana de Biodiversidad, 83, 246-257.

Chávez-Tovar, J. C. (2005). Puma concolor (Linnaeus, 1771). En G. Ceballos y G. Oliva (Coords.), Los mamíferos silvestres de México (pp. 364-367). México, D.F.: Comisión Nacional para el Conocimiento y Uso de la Biodiversidad, Fondo de Cultura Económica.

Currier, M. J. P. (1983). Felis concolor. Mammalian Species, 200, 1-7.

Di Bitetti, M. S., de Angelo, C. D., di Blanco, Y. E. y Paviolo, A. (2010). Niche partitioning and species coexistence in a Neotropical felid assemblage. Acta Oecologica, 36, 403-412.

Emmons, L. H. (1987). Comparative feeding ecology of felids in a Neotropical rainforest. Behavioral Ecology and Sociobiology, 20, 271-283.

Escalante, T., Rodríguez, G. y Morrone, J. J. (2005). Las provincias biogeográficas del componente mexicano de montaña desde la perspectiva de los mamíferos continentales. Revista Mexicana de Biodiversidad, 76, 199-205.

Ferrari-Pérez, F. (1886). Catalogue of animals collected by the geographical and exploring Commission of the Republic of Mexico. Proceedings of the U.S. National Museum, 9, 125-199.

Foster, R. J. y Harmsen, B. J. (2012). A critique of density estimation from camera-trap data. The Journal of Wildlife Management, 76, 224-236.

Foster, V. C., Sarmento, P., Sollmann, R., Tôrres, N., Jàcomo, A. T. A., Negroes, N., et al. (2013). Jaguar and puma activity patterns and predator-prey interactions in four Brazilian biomes. Biotropica, 45, 373-379.

Harmsen, B. J., Foster, R. J., Silver, S. C., Ostro, L. E. T. y Doncaster, C. P. (2009). Spatial and temporal interactions of sympatric jaguars (Panthera onca) and pumas (Puma concolor) in a Neotropical forest. Journal of Mammalogy, 90, 612-620.

Holbrook, J. D., Caso, A., Deyoung, R. W. y Tewes, M. E. (2013). Population genetics of jaguarundis in Mexico: implications for future research and conservation. Wildlife Society Bulletin, 37, 336-341.

Iglesias, J., Sánchez-Cordero, V., Magaña-Cota, G., Bolaños, R., Aranda, M., Hernández, R., et al. (2008). Noteworthy records of margay, Leopardus wiedii and ocelot, Leopardus pardalis in the state of Guanajuato, Mexico. Mammalia, 72, 347-349.

Johnson, W. E., Eizirik, E., Pecon-Slattery, J., Murphy, W. J., Antunes, A., Teeling, E., et al. (2006). The late Miocene radiation of modern Felidae: a genetic assessment. Science, 311, 73-77.

Kéry, M. (2011). Species richness and community dynamics: a conceptual framework. En A. F. O'Connell, J. D. Nichols y K. U. Karanth (Eds.), Camera traps in animal ecology: methods and analyses (pp. 207-231). New York: Springer.

Lira-Torres, I. y Briones-Salas, M. (2012). Abundancia relativa y patrones de actividad de los mamíferos de los Chimalapas, Oaxaca, México. Acta Zoológica Mexicana, 28, 566-585.

Monroy-Vilchis, O., Rodríguez-Soto, C., Zarco-González, M. y Urios, V. (2009). Cougar and jaguar habitat use and activity patterns in central Mexico. Animal Biology, 59, 145-157.
Monroy-Vilchis, O., Zarco-González, M. M., Rodríguez-Soto, C., Soria-Díaz, L. y Urios, V. (2011). Fototrampeo de mamíferos en la sierra Nanchititla, México: abundancia relativa y patrón de actividad. Revista de Biología Tropical, 59, 373-383.

Monterrubio-Rico, T. C., Charre-Medellín, J. F., Zavala-Paramo, M. G., CanoCamacho, H., Pureco-Rivera, M. Q. y León-Paniagua, L. (2012). Evidencias fotográfica, biológica y genética de la presencia actual de jaguaroundi (Puma yagouaroundi) en Michoacán, México. Revista Mexicana de Biodiversidad, $83,825-833$.

Núñez, R., Miller, B. y Lindzey, F. (2000). Food habits of jaguars and pumas in Jalisco, Mexico. Journal of Zoology, 252, 373-379.

Oliveira, T. G. (1998a). Herpailurus yagouaroundi. Mammalian Species, 578, $1-6$.

Oliveira, T. G. (1998b). Leopardus wiedii. Mammalian Species, 579, 1-6.

Porras-Murillo, L. P., Sarmiento-Aguilar, R., Naranjo, E. J. y Vázquez, L. B. (2011). Conservation effectiveness of protected areas in Mexico: effects on medium and large mammals at local and regional scales. International Journal of Biodiversity and Conservation, 3, 487-496.

Ramírez-Bravo, O. E., Bravo-Carrete, E., Hernández-Santín, C., SchinkelBrault, S. y Kinnear, C. (2010). Ocelot (Leopardus pardalis) distribution in the state of Puebla, Central México. Therya, 1, 111-120.

Ramírez-Bravo, O. E., Schinkel-Brault, S. y Hernández-Santín, C. (2010). Nuevo registro del ocelote (Leopardus pardalis) para el estado de Puebla. Therya, 1, 91-94.

Ramírez-Pulido, J., González-Ruiz, N. y Genoways, H. H. (2005). Carnivores from the Mexican state of Puebla: distribution, taxonomy, and conservation. Mastozoología Neotropical, 58, 37-52.

Ramos-Robles, M., Gallina, S. y Mandujano, S. (2013). Habitat and human factors associated with white-tailed deer density in the tropical dry forest of Tehuacán-Cuicatlán Biosphere Reserve, Mexico. Tropical Conservation Science, 6, 70-86.

Reid, F. A. (1997). A field guide to the mammals of Central America and Southeast Mexico. New York: Oxford University Press.

Rzedowski, J. (1978). Vegetación de México. México, D.F.: Limusa.

Semarnat (Secretaría de Medio Ambiente y Recursos Naturales). (2010). Norma Oficial Mexicana NOM-059-SEMARNAT-2010, Protección ambientalEspecies nativas de México de flora y fauna silvestres-Categorías de riesgo y especificaciones para su inclusión, exclusión o cambio-Lista de especies en riesgo. Diario Oficial de la Federación. 30 de diciembre de 2010, Segunda Sección. México.

Tewes, M. E. y Everett, D. D. (1982). Status and distribution of the endangered ocelot and jaguarundi in Texas. En S. D. Miller y D. D. Everett (Eds.), Cats of the world: biology, conservation and management (pp. 147-158). Washington, D.C: National Wildlife Federation.

Tobler, M. W., Carrillo-Percastegui, S. E., Leite-Pitman, R., Mares, R. y Powell, G. (2008). An evaluation of camera traps for inventorying large and medium sized terrestrial rainforest mammals. Animal Conservation, 11, 169-178.

Valenzuela-Galván, D. y Vázquez, L. B. (2008). Prioritizing areas for conservation of Mexican carnivores considering natural protected areas and human population density. Animal Conservation, 11, 215-223.

Valenzuela-Galván, D., de León-Ibarra, A., Lavalle-Sánchez, A., Orozco-Lugo, L. y Chávez, C. (2013). The margay Leopardus wiedii and bobcat Lynx rufus from the dry forests of Southern Morelos, Mexico. The Southwestern Naturalist, 58, 118-120.

Valiente-Banuet, A., Casas, A., Alcántara, A., Dávila, P., Flores-Hernández, N., Arizmendi, M. C., et al. (2000). La vegetación del valle de TehuacánCuicatlán. Boletín de la Sociedad Botánica de México, 67, 27-74. 